\title{
Tumor del estroma gastrointestinal como causa de hemorragia del tubo digestivo: presentación de un caso
}

\author{
Gastrointestinal stromal tumor as a cause of gastrointestinal bleeding: \\ presentation of a case
}

Julio César López-Valdés, ${ }^{*}$ Willy Nava-Gutiérrez, ${ }^{* *}$ Alejandra Astaburuaga-Gómez, ${ }^{* *}$ Jaime Eduardo Pérez-Perales***

Palabras clave:

Tumor del

estroma gástrico,

hematemesis,

sangrado del tubo

digestivo, imatinib.

Key words: Gastric

stromal tumor,

hematemesis,

gastrointestinal bleeding, imatinib.
* Jefatura de Enseñanza e Investigación, ISSSTE, México, DF, México.

** Facultad de Medicina de Tampico "Dr. Alberto Romo Caballero" de la Universidad Autónoma de Tamaulipas, Tampico, Tamaulipas, México. *** Miembro de la Asociación Mexicana de Cirugía General.

Recibido: 07/01/2015 Aceptado: 18/03/2016

\section{RESUMEN}

Introducción: Los tumores del estroma gastrointestinal se ubican entre las neoplasias mesenquimales más frecuentes del aparato gastrointestinal; representan del 0.1-3\% del total. Sus principales localizaciones son el estómago y el intestino delgado (70-90\%). La mayoría de estos tumores son asintomáticos; su diagnóstico generalmente es incidental. Sin embargo, en algunos casos su principal manifestación es la hemorragia del tubo digestivo en forma de hematemesis, muchas veces considerada como "de origen desconocido". Descripción del caso: Varón de 55 años con antecedentes de diabetes mellitus tipo 2 e hipertensión arterial, con historia de enfermedad por reflujo gastroesofágico y gastritis, que acude al servicio de urgencias por dolor abdominal difuso, plenitud postprandial y varios episodios de hematemesis. Los análisis de laboratorio arrojaron los siguientes datos: hemoglobina $9.3 \mathrm{~g} / \mathrm{dL}$, hematocrito $28.5 \%$, plaquetas $324 \times 103$, leucocitos $11.5 \times 103$, glucosa $183 \mathrm{mg} / \mathrm{dL}$, creatinina $1.1 \mathrm{mg} /$ dL, albúmina $4.4 \mathrm{~g} / \mathrm{dL}$. La gastroscopia y la tomografía contrastada revelaron una masa subepitelial de bordes definidos localizada en la curvatura mayor del cuerpo del estómago, de cinco $\mathrm{cm}$ de diámetro, con datos sugestivos de ulceración. Se realizó una resección de la misma con márgenes libres acompañada de terapia inmunomoduladora a base de imatinib. Se logró la remisión total año y medio después de iniciado el tratamiento.

\section{ABSTRACT}

Introduction: Gastrointestinal stromal tumors are the most frequent mesenchymal neoplasms of the gastrointestinal tract, representing 0.1 to $3 \%$ of all gastrointestinal neoplasms. These tumors are mainly located in the stomach and small intestine (70 to 90\%). Most of them are asymptomatic and are mostly incidental findings. However, there is a group of cases in which the main manifestation is gastrointestinal bleeding (hematemesis), which is often regarded as being "of unknown origin". Case description: A 55-year old male, with a medical history of type 2 diabetes and arterial hypertension, gastro-oesophageal reflux disease and gastritis. He was seen in the hospital with diffuse abdominal pain, postprandial fullness, and episodes of hematemesis. Laboratory findings showed, hemoglobin $9.3 \mathrm{~g} / \mathrm{dL}$, hematocrit $28.5 \%$, platelets $324 \times 103$, white cells $11.5 \times 103$, glucose $183 \mathrm{mg} / \mathrm{dL}$, creatinine $1.1 \mathrm{mg} / \mathrm{dL}$, and albumin $4.4 \mathrm{~g} / \mathrm{dL}$. Contrasted CT and gastroscopy revealed a sub-epithelial mass with defined borders located on the greater curvature of the gastric body, 5-6 cm in diameter, and signs suggestive of ulceration. $R 0$ resection was performed accompanied by immunomodulatory therapy with imatinib, bringing the total remission a year and a half after starting treatment.

\section{INTRODUCCIÓN}

T os tumores del estroma gastrointestinal $\mathcal{L}$ (GIST) son neoplasias cuyo origen es el mesénquima del tracto digestivo (células intersticiales de Cajal). Pertenecen a la familia denominada "sarcomas de tejidos blandos". A pesar de que son poco frecuentes, constituyen la forma más común de tumores mesenquimales del tracto gastrointestinal. Aunque es posible encontrarlos en toda la extensión del aparato digestivo, el $70-90 \%$ de ellos se encuentra en el estómago y el intestino delgado. ${ }^{1,2}$ Se estima que existen de 10 a 20 casos por millón de habitantes, de los cuales el $20-30 \%$ son potencialmente malignos, además de poseer una alta tasa de recidivas (> $30 \%$ ). Su edad pico de incidencia se sitúa entre la sexta y séptima década de vida (55-65 años); 
tienen una ligera predilección por el género masculino y generalmente son asintomáticos. Cuando dan manifestaciones clínicas, éstas suelen ser tan inespecíficas que hacen difícil el diagnóstico. En años recientes se observó que pueden ser una causa de hemorragia del tubo digestivo (HTD) "de origen desconocido". ${ }^{2-7}$

A continuación presentamos el caso de un individuo cuya manifestación principal fue la hematemesis de repetición.

\section{CASO CLÍNICO}

Hombre de 55 años de edad con hipertensión arterial de dos años de evolución tratada con amlodipino y valsartán (5 y 160 mg/24 horas, respectivamente) y diabetes mellitus tipo 2 de seis meses de evolución tratada con metformina (850 mg/24 horas).

Al interrogatorio, el paciente refirió datos sugerentes de enfermedad por reflujo gastroesofágico desde hacía cuatro años. Tomó omeprazol a libre demanda. Indicó ser consumidor crónico de alcohol y llevar una dieta rica en alimentos grasos e irritantes.

Ingresó al Servicio de Urgencias el 7 de febrero de 2013 por hematemesis en posos de café no cuantificada, acompañada de malestar general, debilidad muscular, dolor abdominal difuso y plenitud postprandial, sin pérdida de peso. A las pocas horas de su ingreso repitió el cuadro de hematemesis seguido de una evacuación meléni- ca. Se inició reposición de líquidos y electrolitos por vía intravenosa. También se realizó lavado gástrico por medio de sonda nasogástrica.

Exploración física: peso 107 kg, talla $168 \mathrm{~cm}$, escasa presencia de vello corporal, palidez general sin ictericia. Tórax con leve ginecomastia, con ruidos respiratorios normales, sin adenomegalias en fosas supraclaviculares ni en las axilas.

Abdomen simétrico y globoso a expensas de grueso panículo adiposo, con cicatriz umbilical central, con dolor generalizado a la palpación media-profunda de predominio en hipocondrio izquierdo, con masa perceptible a la palpación profunda en el hipocondrio izquierdo.

Datos de laboratorio: hemoglobina $9.3 \mathrm{~g} /$ $\mathrm{dL}$, hematocrito $28.5 \%$ plaquetas $324 \times 103$, leucocitos $11.5 \times 103$, glucosa $183 \mathrm{mg} / \mathrm{dL}$, creatinina $1.1 \mathrm{mg} / \mathrm{dL}$, albúmina $4.4 \mathrm{~g} / \mathrm{L}$, globulina $3.4 \mathrm{~g} / \mathrm{L}$, bilirrubina directa $0.1 \mathrm{mg} / \mathrm{dL}$, bilirrubina indirecta $0.4 \mathrm{mg} / \mathrm{dL}$, fosfatasa alcalina 67 U/L, transaminasa glutámico pirúvica $190 \mathrm{U} / \mathrm{L}$, transaminasa glutámico oxalacética $136 \mathrm{U} / \mathrm{L}$.

A los dos días del ingreso se le practicó gastroscopia que reveló una masa subepitelial de bordes bien definidos localizada en la curvatura mayor del cuerpo gástrico. No se tomó biopsia por temor a desencadenar un nuevo episodio hemorrágico o diseminación tumoral.

Al día siguiente se llevó a cabo tomografía computada (TC) contrastada en la que se observó una tumoración ulcerada de 5-6 cm de diámetro en el fundus gástrico (Figura 1).

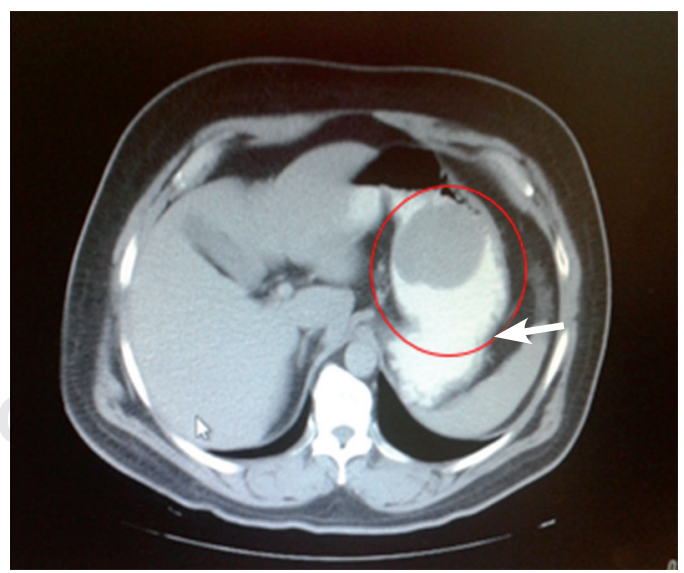

Figura 1. Tomografia computada contrastada que muestra imagen hipodensa circular de bordes bien definidos, de aproximadamente 5-6 cm de diámetro mayor, compatible con masa ocupativa en fundus gástrico con datos de ulceración (flecha blanca). 
Se ejecutó resección quirúrgica con márgenes libres por abordaje abierto, con conservación de la curvatura menor, y se obtuvo el diagnóstico de tumor de la pared gástrica (GIST) confirmado por el Departamento de Patología.

Se indicó imatinib 100 mg cada 12 horas por nueve semanas; después se incrementó la dosis a $200 \mathrm{mg}$ cada 12 horas. A lo largo del seguimiento clínico, el paciente presentó náuseas, mareo, fatiga y diarrea que cedieron después del primer mes de tratamiento.

En octubre del mismo año, se le realizó tomografía computada por emisión de positrones (PET) (Figura 2); no se detectaron datos de actividad tumoral en el sistema digestivo, pulmones, cerebro y ganglios linfáticos.

El paciente continuó bajo tratamiento con imatinib. El plan fue completar el ciclo de un año de tratamiento.

El día 29 de agosto de 2014 le fue practicada gastroscopia con la finalidad de observar su evolución. No mostró anomalías, con resultados de la biopsia normales.

\section{DISCUSIÓN}

La concepción de los GIST ha implicado innumerables controversias, ya que en el pasado han sido erróneamente clasificados como tumores de células musculares lisas (leiomiomas, leiomioblastomas y leiomiosarcomas) $u$ otros derivados mesenquimatosos. Con el advenimiento de la inmunohistoquímica, el
GIST se definió como una neoplasia derivada de células madre mesenquimales pluripotenciales programadas para diferenciarse en células intersticiales de Cajal positivas al marcador KIT (CD117) y PDGFRA. 2,6-11

Debido a su amplia distribución, la mayor parte de estos tumores se diagnostica de manera fortuita en estudios rutinarios de imagen o durante alguna cirugía. Los sitios más comunes de localización son el estómago (50-60\%) y el intestino delgado (30\%). La frecuencia para el esófago es del $5 \%$ y para el colon, de menos del 5\%; excepcionalmente se encuentran en la pared abdominal, el omento, el mesenterio y el retroperitoneo. ${ }^{1,2,6,12,13}$

El diagnóstico es difícil debido a su presentación clínica variable. Con gran frecuencia, el tumor es silente hasta que adquiere un tamaño considerable, momento en el que comienza a producir síntomas inespecíficos muchas veces dependientes del sitio de la lesión, los cuales pueden ser náuseas, vómito, pérdida de peso, masa palpable, plenitud postprandial o alteración del hábito intestinal. ${ }^{4,13-15}$ Sin embargo, como se evidenció en este caso, existe un grupo de pacientes en los que la principal manifestación es hemorragia del tubo digestivo causada por la ulceración del tumor. La hemorragia del tubo digestivo por GIST representa el 1\% del total de las hemorragias del tubo digestivo y es ocasionada por erosión de la mucosa adyacente debido al efecto de masa; ${ }^{16}$ aunque su frecuencia no es del todo clara, existen informes
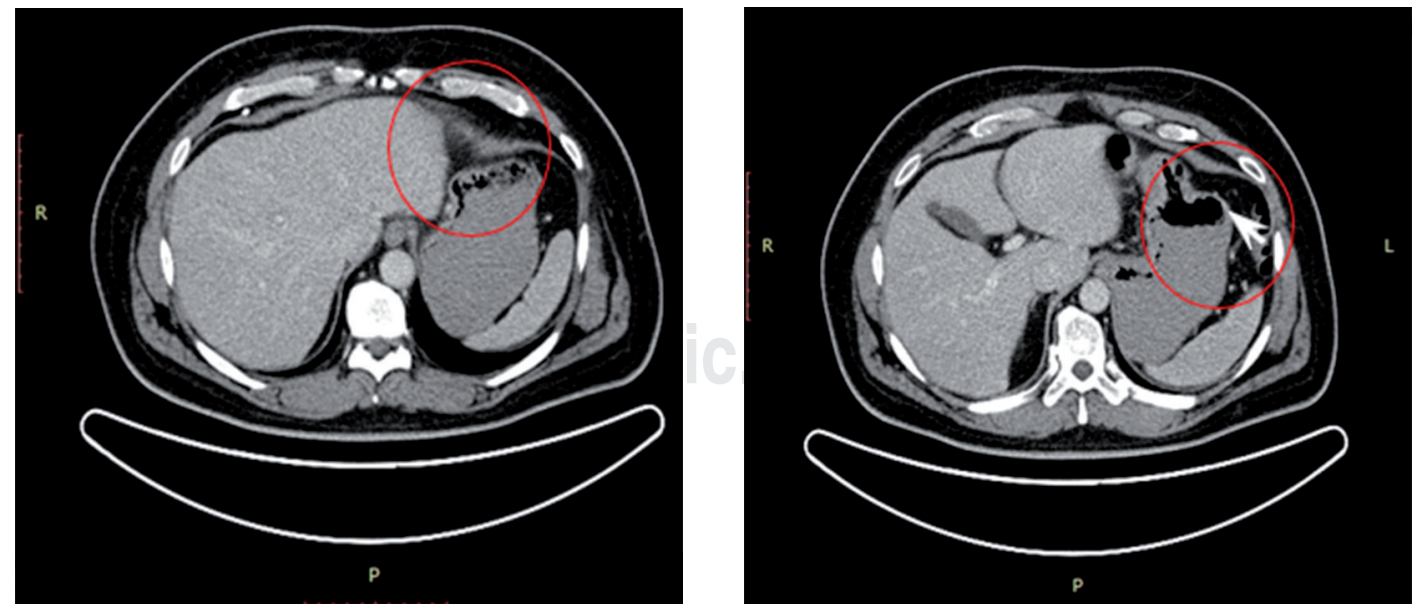

Figura 2. Imagen de control seis meses después de la resección quirúrgica (PET-TC). 
que sugieren su presencia hasta en $60 \%$ de los casos clínicos.

Novitsky y colaboradores ${ }^{17}$ observaron anemia en el $20 \%$ de los individuos, así como hemorragia masiva en $30 \%$ de los casos al momento del diagnóstico. Braghetto y su grupo ${ }^{18}$ informaron una frecuencia de hemorragia del tubo digestivo de $54.4 \%$, manifestada como anemia, melena o hematemesis. Nuestro paciente presentó todas estas manifestaciones.

En nuestro caso, la hemorragia del tubo digestivo y el dolor abdominal crónico fueron las manifestaciones de la enfermedad y por las cuales nuestro paciente acudió al Servicio de Urgencias. Tanto la gastroscopia como la tomografía computada revelaron lesiones erosivas en la mucosa cercana al tumor, lo cual concuerda con los informes y series de casos previos. En cuanto a los estudios de imagen, los datos obtenidos son similares en todas las series e informes, incluido el nuestro. La tomografía computada es el método de elección para el diagnóstico, así como para obtener la localización del tumor, la extensión local y la evaluación del tamaño. Los hallazgos habituales suelen ser grandes masas bien delineadas, hiperdensas, de aspecto heterogéneo, con componente exofítico e intraluminal poliploide que otorga una apariencia en "reloj de arena". Al emplear medio de contraste ocurre magnificación y reforzamiento de los bordes. ${ }^{1,4,19-22}$

Otros posibles hallazgos son áreas de atenuación de menor tamaño dentro o en la periferia de la lesión secundarias a necrosis, erosión o hemorragia. ${ }^{1,20}$ Existen informes en donde se menciona que el ultrasonido endoscópico tiene una sensibilidad del 95\% y una especificidad del $72 \%$, cifras mayores a las obtenidas mediante tomografía computada, aunque demostró ser inservible para el diagnóstico de lesiones no GIST (56\%), por lo cual sólo debe ser considerado ante una alta sospecha de este tipo de neoplasias. ${ }^{22,23}$

Para el diagnóstico histopatológico, la biopsia guiada es la de mayor éxito; sin embargo, usualmente es solo a través de la resección quirúrgica como puede obtenerse tejido suficiente para su confirmación. Además, la resección quirúrgica no sólo representa un método diagnóstico sino terapéutico y ayuda a establecer el pronóstico. ${ }^{15,22}$
En este caso, aunque en un principio se consideró como un fibroma, el diagnóstico de certeza se obtuvo mediante el estudio de histopatología, el cual reveló un GIST de bajo grado de malignidad conformado por una lesión en la pared gástrica de $4.5 \times 3 \times 0.5$ $\mathrm{cm}$, con lesión nodular de serosa a submucosa. Asimismo, se confirmó a través de los estudios de inmunohistoquímica complementarios, que arrojaron marcadores positivos propios de este tipo de neoplasias (CD117/c KIT).

El tratamiento de elección es el quirúrgico y dependerá de diversos factores como la localización, el tamaño, las manifestaciones y las complicaciones; es decir, es necesario individualizar el abordaje quirúrgico en cada caso. ${ }^{15}$

Como sucede con cualquier neoplasia, la resección quirúrgica con bordes libres (R0) es la técnica a emplear en aquellos casos con evidencia de enfermedad localizada y ausencia de metástasis. Puesto que estos tumores no suelen infiltrar de forma difusa la pared del estómago, basta con la resección de 1-2 cm de bordes libres de tumor. ${ }^{4,15,23-28}$

La conveniencia de la cirugía laparoscópica en los GIST confinados al estómago ha causado controversia debido a que la extensión de los márgenes libres de tumor o la linfadenectomía no se han asociado con mejora de los resultados oncológicos, por lo que muchos centros se han dedicado a investigar la factibilidad y seguridad de un abordaje mínimamente invasivo en el tratamiento de los GIST. ${ }^{29}$ Existen series de tratamiento laparoscópico que demostraron una mayor seguridad con menor tiempo de recuperación y trauma, lo que conlleva a una reducción de costos. Al igual que la resección abierta, la técnica laparoscópica debe lograr la resección extracapsular del tumor intacto y la conservación del tejido gástrico normal, evitando la siembra tumoral. ${ }^{23}$

En nuestro caso, se realizó la extirpación del tumor de manera abierta, llevando a cabo una resección con bordes libres de lesión (R0).

La linfadenectomía rara vez se justifica, ya que las metástasis a nodos linfáticos son infrecuentes; aun así, el riesgo de recurrencia alcanza $27-84 \%$, con tasas de supervivencia a cinco años de $40-55 \%$. $^{2,14,15,23-25,27}$

La quimioterapia y la radioterapia convencionales son poco eficaces. Recientemente, se 
introdujo el mesilato de imatinib (STI571), un agente oral derivado de la 2-fenilaminopirimidina, inhibidor selectivo del receptor kit de la tirosincinasa, aprobado para el tratamiento de GIST metastásicos o "no resecables". Este medicamento ha demostrado resultados benéficos incluso al ser empleado como adyuvante postquirúrgico. Así ocurrió en nuestro caso. ${ }^{4,13,24-26,30-32}$

En caso de existir resistencia al imatinib, se recomienda el uso de sunitinib. $\mathrm{Al}$ igual que el primero, es un inhibidor de la tirosincinasa de focalización KIT y es también un agente antiangiogénesis que trabaja con los receptores del factor de crecimiento derivados de plaquetas $\alpha$ y $\beta$ y el factor de crecimiento endotelial vascular. ${ }^{4,33,34}$

A pesar de la eficacia de estos fármacos, existen recidivas tumorales, por lo cual ha sido propuesto como tercera línea de tratamiento el regorafenib. Es un inhibidor multicinasa con actividad contra KIT, PDGFR y el factor de crecimiento endotelial vascular que tiene buenos resultados en estos casos y pocos efectos adversos, tales como hipertensión arterial (23\%), reacción cutánea mano-pie $(20 \%)$ y diarrea (5\%). ${ }^{2,35}$

Debido a su comportamiento clínico insidioso, resulta común que los tumores GIST pasen inadvertidos en etapas tempranas. Todos los GIST son considerados potencialmente malignos.

En caso de presentar metástasis, se desarrollan de 10 a 15 años después de la cirugía primaria y se sitúan comúnmente en la cavidad abdominal y el hígado; en menor medida en la pleura y los pulmones. ${ }^{36}$

La tomografía por emisión de positronestomografía computada (PET-TC) corporal constituye el método de evaluación a la respuesta terapéutica con los inhibidores de tirosincinasa. ${ }^{37}$

Las complicaciones más comunes de los GIST son intususcepción o invaginación intestinal, obstrucción intestinal, colestasis por compresión de la vía biliar y hemorragia masiva debido a ulceración del tumor. ${ }^{1,2,4,26}$

\section{CONFLICTO DE INTERESES}

Los autores declaran no tener ningún conflicto de intereses.

\section{REFERENCIAS}

1. Cano-Muñoz I, Avendaño-Avalos DB, Cuervo-Pérez L. Tumores del estroma gastrointestinal: revisión por imagen y correlación anatomopatológica. Presentación de cuatro casos. Ana Rad Mex. 2011; 1: 48-59.

2. Rammohan A, Sathyanesan J, Rajendran K, et al. A gist of gastrointestinal stromal tumors: A review. World J Gastroinstest Oncol. 2013; 5: 102-112.

3. Nemes C, Rogojan L, Surdea-Blaga T, et al. Gastrointestinal stromal tumor (GIST) associated with synchronous colonadenocarcinoma: A case report. J Gastrointestin Liver Dis. 2012; 21: 101-103.

4. Acin-Gandara D, Pereira-Pérez F, Castaño-Pascual A, et al. Tumores estromales gastrointestinales: diagnóstico y tratamiento. Cir Ciruj. 2012; 80: 44-49.

5. Shukla S, Singh SK, Pujani M. Multicentric malignant gastrointestinal stromal tumor. Saudi J Gastroenterol. 2009; 15: 45-48.

6. Chapa-Azuela O, Ortiz-Higareda V, Etchegaray-Dondé A, et al. Tumores del estroma gastrointestinal (GIST) ampulares tratados con pancreaticoduodenectomía. Reporte de dos casos y revisión de la literatura. Rev Med Hosp Gen Mex. 2013; 76: 52-56.

7. Pila-Pérez R, Rosales-Torres MD, Pila-Peláez R, et al. Tumor del estroma gastrointestinal (GIST): descripción de una rara observación. Rev Col Gastroenterol. 2009; 24: 403-407.

8. Arroyo-Martínez L, Álvarez-Pertuz H, Acuña-Calvo J. Tumor gástrico estromal como causa de sangrado digestivo. Acta Med Costarric. 2006; 48: 131-134.

9. Xie R. Gastrointestinal stromal tumors: short review on KIT/PDGFRA gene mutations and molecular therapy. J Gastroint Dig Syst. 2013; S12: 004.

10. Fletcher CDM, Berman JJ, Corless C, et al. Diagnosis of gastrointestinal stromal tumors: A consensus approach. Int J Surg Pathol. 2002; 10: 81-89.

11. Chi $P$, Chen $Y, Z$ hang $L$, et al. ETV1 is a lineage survival factor that cooperates with KIT in gastrointestinal stromal tumours. Nature. 2010; 467: 849-855.

12. Soto-GS, del Pozo-LM, Kuschel-HC, et al. Tumores estromales del tubo digestivo (GIST). A propósito de un caso clínico. Cuad Cir. 2003; 17: 37-42.

13. García-Marín A, Matín-Gil J, Nofuentes-Riera C, et al. Hemorragia digestiva baja secundaria a tumor del estroma gastrointestinal rectal. Rev Gastroenterol Mex. 2010; 75: 332-334.

14. Neira J, Morán C, Correa R, et al. Tumor del estroma gastrointestinal, en duodeno, como causa de hemorragia. Rev Med FCM-UCSG. 2011; 17: 274-279.

15. Fernández JÁ, Sánchez-Cánovas ME, Parrilla P. Controversias en el tratamiento quirúrgico de los tumores del estroma gastrointestinal (GIST) primarios. Cir Esp. 2010; 88: 69-80.

16. Bucher P, Villiger P, Egger JF, et al. Management of grastrointestinal stromal tumours: From diagnosis to treatment. Swiss Med Wkly. 2004; 134: 145-153.

17. Novitsky YW, Kercher KW, Sing RF. Long-term outcomes of laparoscopic resection of gastric gastrointestinal stromal tumors. Ann Surg. 2006; 243: 738-747.

18. Braghetto I, Parada FJ, Cardemil G. Tumores gastrointestinales estromales (GIST): Experiencia del Servicio de Cirugía de la Universidad de Chile entre 1999 y 2005. Rev Med Chile. 2007; 135: 551-557. 
19. Gómez G, Lombo M, Peñaloza F. Tumores estromales gástricos (GIST) malignos. Presentación de dos casos. Rev Colomb Gastroenterol. 2004; 19: 137-142.

20. Oyanedel QR, O'Brien SA, Pizarro-GA, et al. Tumor estromal gastrointestinal (GIST): formas de presentación. Rev Chil Radiol. 2005; 11: 13-18.

21. Bannura CG, Cornejo CV. Hemorragia digestiva baja recurrente secundaria a GIST de yeyuno. Rev Chil Cir. 2011; 63: 631-634.

22. Bahena-Aponte JA, González-Contreras QH. Hemorragia de tubo digestivo alto como presentación inicial de tumor del estroma gastrointestinal a nivel de yeyuno proximal. Reporte de caso. Rev Invest Med Sur Mex. 2011; 18: 60-63.

23. Shu ZB, Sun LB, Li JP, et al. Laparoscopic versus open resection of gastric gastrointestinal stromal tumors. Chin J Cancer Res. 2013; 25: 175-182.

24. Daigle C, Meneghetti AT, Lam J, et al. Laparoscopic management of gastrointestinal stromal tumours: review at a Canadian centre. Can J Surg. 2012; 55: 105-109.

25. DeMatteo RP, Heinrich MC, El-Rifai WM, et al. Clinical management of gastrointestinal stromal tumors: before and after STI-571. Human Pathol. 2002; 33: 466-477.

26. Cappellani A, Piccolo G, Cardi F, et al. Giant gastrointestinal stromal tumor (GIST) of the stomach cause of high bowel obstruction: surgical management. World J Surg Oncol. 2013; 11: 172.

27. Mouaqit O, Chbani L, Maazaz K, et al. A large gastrointestinal stromal tumor of the duodenum treated by partial duodenectomy with Roux-en-Y duodenojejunostomy: A case report. J MedCase Reports. 2013; 7: 184.

28. Germano-Bassi D, Pérez-Adorno-da Silva F, de Oliveira-Sousa FM. GIST-induced upper gastrointestinal bleeding. Rev Assoc Med Bras. 2010; 56: 520-521.
29. DeMatteo RP, Lewis JJ, Leung D, et al. Two hundred gastrointestinal stromal tumors: Recurrence patterns and prognostic factors for survival. Ann Surg. 2000; 231: 51-58.

30. Parikh PM, Gupta S. Management of gastrointestinal stromal tumor: The imatinib era and beyond. Indian J Cancer. 2013; 50: 31-40.

31. Biasco G, Velo D, Angriman I, et al. Gastrointestinal stromal tumors: Report of an audit and review of literature. Eur J Cancer Prev. 2009; 18: 106-116.

32. Demetri GD, von Meheren M, Blanke CD, et al. Efficacy and safety of imatinib mesylate in advanced gastrointestinal stromal tumors. N Engl J Med. 2002; 347: 472-480.

33. Bracci R, Maccaroni E, Cascinu S. Transient sunitinib resistance in gastrointestinal stromal tumors. $\mathrm{N}$ Engl J Med. 2013; 368: 2042-2043.

34. Lamba G, Ambrale S, Lee B, et al. Recent advances and novel agents for gastrointestinal stromal tumor (GIST). J Hematol Oncol. 2012; 5: 21.

35. Demetri GD, Reichardt P, Kang Y, et al. Efficacy and safety of regorafenib for advanced gastrointestinal stromal tumours after failure of imatinib and sunitinib: An international, multicentre, prospective, randomised, placebo-controlled phase 3 trial (GRID). Lancet. 2013; 381: 295-302.

36. Poveda A, Artigas V, Casado A, et al. Guía de práctica clínica en los tumores estromales gastrointestinales (GIST): actualización 2008. Cir Esp. 2008; 84: 1-21.

37. Alberto S, Sánchez P, Oliveira M, et al. Tumores del estroma gastrointestinal. Estudio retrospectivo de 43 casos. Rev Esp Enferm Dig. 2008; 100: 696-700.

\section{Correspondencia: \\ Julio César López Valdés}

Tels: 5559029446 / 8332949489

E-mail: jc.lopz@live.com 